\title{
ON ASYMPTOTIC VOLUME OF FINSLER TORI, MINIMAL SURFACES IN NORMED SPACES, AND SYMPLECTIC FILLING VOLUME.
}

\author{
D. BURAGO AND S. IVANOV
}

\section{INTRODUCTION}

The main "unconditional" result of this paper, Theorem 3, states that every two-dimensional affine disc in a normed space (that is, a disc contained in a two-dimensional affine subspace) is an area minimizing surface among all immersed discs with the same boundary, with respect to the symplectic (Holmes-Thompson) surface area. To emphasize that this is not at all obvious, it may be worth mentioning that a similar statement with rational chains in place of immersed discs is incorrect (Theorem 2), and that it is not known for surfaces that may not be topological discs. The result still may not sound too exciting to the reader who never looked at the problem before, even though the problem goes back to Busemann's works in the 50th (see [BES], [Th] and references there), and the proof heavily relies on asymptotic geometry of tori. We belive that it is more important that we embed this problem into a whole area of (mostly open) problems, as well as give some partial results and suggest certain directions of how attack them.

We begin with a trivial statement:

Minimality of Flats in Euclidean Spaces. A ball in an $n$-dimensional affine subspace of a Euclidean space has the smallest $n$-dimensional surface area among all $n$-dimensional immersed discs with the same boundary.

The proof boils down to considering the orthogonal projection onto the affine subspace, which is an area non-increasing map. Of course, the statement remains true (and obvious) if "immersed discs" are substituted by "immersed surfaces" or "Lipschits singular chains with real coefficients".

The next statement is an easy corollary of Besicovitch's inequality:

Riemannian Filling Volume in Euclidean Spaces. The Euclidean volume of a bounded region in a Euclidean space is less than or equal to the volume of this region with respect to any Riemannian metric whose distances between boundary points majorize those of the Euclidean distance function.

Now let us formulate a volume growth theorem which is the main result of [BI1] and served as a starting point for this research.

The first author is partially supported by an Alferd P. Sloan Fellowship and NSF Grant DMS-9803129. The second author is supported by RFBR grants 99-01-00104 and 00-15-96024. 
Volume Growth of Riemannian Periodic Metrics. Let $(M, g)$ be a universal cover of a Riemannian $n$-torus. One easily sees that the volume of a large metric ball of radius $r \rightarrow \infty$ in $(M, g)$ is $C(M, g) \cdot r^{n}+o\left(r^{n}\right)$, for a positive constant $C(M, g)$. The volume growth theorem asserts that

$$
C(M, g) \geq C\left(\mathbf{R}^{n}, \text { standard metric }\right) .
$$

These three facts are closely related. First, there are obvious implications: volume growth theorem $\Rightarrow$ filling volume inequality $\Rightarrow$ minimality of flats.

On the other hand, the argument proving the volume growth theorem is based on embedding $(M, g)$ into an appropriate Banach space $V^{N}$. Then, by a (rather tricky) choice of an auxiliary Euclidean structure in this Banach space, the theorem is reduced to just the Minimality of Flats in Euclidean Spaces, which is trivial.

One notices that both the target space for the embedding and the embedded surface arising in the proof of the volume growth theorem carry natural Banach (resp., Finsler) structures. The main difficulty in the proof is converting these spaces into appropriate Euclidean (Riemannian) ones. On the other hand, one naturally formulates analogs of the assertions for Banach (Finsler) spaces. It turns out that it is even easier to show the equivalence of these assertions to each other than for their Euclidean (Riemannian) counterparts, but neither of them is obvious any more. We formulate them as the following three problems:

Minimality of Flats in Banach Spaces (Busemann, Thompson; see [Th] and references there): Does a ball in an $n$-dimensional affine subspace of a Banach space minimize volume among all $n$-dimensional surfaces with the same boundary?

Finsler Filling Volume Problem: For a bounded region in a finitedimensional Banach space, does its flat Finsler metric have the least volume among all Finsler metric whose distances between boundary points majorize those of the Banach distance function? (Here it is essential that we minimize over Finsler metrics; whereas it seems that assuming that the ambient Banach space is Euclidean does not make much difference.)

Finsler Volume Growth Problem: Does the Volume Growth Theorem hold for Finsler tori? In other words, does the volume of balls in the universal cover of a Finsler torus asymptotically grows at least as fast as that in a Banach space?

In this paper we discuss the relationships between these and several related problems and give a few partial results. Certainly, the formulations above are imprecise (especially the third one, where we have to specify the Banach space to compare with). In order to make the formulations rigorous we need some preliminaries.

\section{Preliminaries}

2.1. Finsler metrics. By a Finsler manifold we mean a smooth manifold $M^{n}$ together with a continuous function $\Phi: T M \rightarrow \mathbf{R}$ such that for every 
$x \in M$, the restriction of $\Phi$ to the tangent space $T_{x} M$ is a vector-space norm, in other words, $\left(T_{x} M,\left.\Phi\right|_{T_{x}} M\right)$ is an $n$-dimensional Banach space.

Notice that, unlike some authors, we consider only Finsler structures given by (symmetric) norms. Riemannian manifolds are Finsler manifolds whose Finsler structure happened to be Euclidean on each fiber of the tangent bundle.

One may impose additional restrictions on $\Phi$ such as being smooth (outside the zero section) and stricly convex (i. e. such that for every $x \in M$ the second derivatives of $\left.\Phi^{2}\right|_{T_{x} M \backslash\{0\}}$ are positive definite). These additional assumptions do not make any difference in the context of this paper. However, we use them in some of our proofs (more general statements then follow by approximation).

¿From metric viewpooint, $\Phi$ enables us to measure length of smooth curves, namely:

$$
\operatorname{Length}(\gamma)=\int \Phi\left(\frac{d \gamma}{d t}\right) d t
$$

This equips $M$ with a distance function: the distance between two points is the infimum of lengths of (piecewise smooth) curves connecting the points. This distance function is denoted by $d_{\Phi}$, and we omit the subscript $\Phi$ if this does not cause ambiguity.

¿From dynamical viewpoint, the function $\Phi^{2}$ (where $\Phi$ is smooth and strictly convex) may be regarded as a Lagrangian, and hence it gives rise to a Lagrangian flow on $T M$. In this context, Finsler manifolds are nothing but Lagrangian systems whose Lagrangians are homogeneous of order 2. Trajectories of these Lagrangian systems are just geodesics on the corresponding Finsler manifolds. In this set-up, Riemannian geodesic flows are Lagrangian flows with quadratic Lagrangians.

Finite-dimensional Banach spaces are trivial examples of Finsler manifolds. Namely, a norm $\|\cdot\|$ on a vector space $V$ defines a Finsler structure $\Phi$ on $V$ by $\Phi(x, v)=\|v\|$ for $x \in V, v \in T_{x} V \cong V$. We say that a Finsler metrics flat if it is (locally) isometric to a Banach space. An (immersed) submanifold of a Banach space naturally carries a Finsler structure defined by restricting the norm to the submanifold's tangent space. In fact, every suficiently smooth Finsler manifold is isometric to a submanifold of a finite-dimensional Banach space ([BI2]).

2.2. Finsler volumes. For a Riemannian manifold, there are two important aspects of Riemannian volume: it is equal to $n$-dimensional Hausdorff measure, and it arises as the projection of Liouville measure from the unit tangent bundle. After converting a Riemannian geodesic flow into a Hamiltonian flow on the co-tangent bundle, this volume can be viewed as a projection of the symplectic volume.

For Finsler manifolds, these features are inherited by two different volumes. The so-called Busemann volume is nothing but $n$-dimensional Hausdorff measure on a Finsler manifold. The other volume, which is sometimes 
called the Holmes-Thompson volume, or symplectic volume, is just defined as the projection of the canonical symplectic volume from the bundle of unit co-tangent balls.

More precisely, for a Finsler manifold $\left(M^{n}, \Phi\right)$ one defines a fiber-wise norm $\Phi^{*}: T^{*} M \rightarrow \mathbf{R}$ so that for every $x \in M$ the restriction $\left.\Phi^{*}\right|_{T_{x}^{*} M}$ is the norm dual to $\left.\Phi\right|_{T_{x} M}$. (Alternatively, one can say that $\frac{1}{2}\left(\Phi^{*}\right)^{2}$ is the Legendre transform of $\frac{1}{2} \Phi^{2}$, thus $\left(\Phi^{*}\right)^{2}$ is the Hamiltonian associated with the Finsler structure.) Then the Holmes-Thompson volume of a region $A \subset M$ equals the symplectic volume of the set $\bigcup_{x \in A}\left\{w \in T_{x}^{*} M: \Phi^{*}(w) \leq 1\right\}$, divided (for normalization) by the Euclidean volume of a unit ball in $\mathbf{R}^{n}$.

In this paper, we will be mainly concerned with the Holmes-Thompson volume. It seems to be more natural in any dynamical set-up, and it is more convenient to operate with.

Notice that there are also notions of volume for Finsler metrics (such as mass and mass* from [Gr]) that essentially substitute a Finsler metric by a certain Riemannian one. Such volumes may be especially designed to work with Jacobians, and for them our problems may easily reduce to their Riemannian counterparts. While such volumes may be extremely useful when working with a Riemannian problem that requires some Finsler considerations, we do not think that they are natural in Finsler geometry. For instance, they are often not sensitive: they can stay unchanged when a metric is increased on an open subset of the tangent bundle.

Finsler volumes can be expressed by integral formulas in coordinates. Namely, if Vol is a Finsler volume functional which depends continuously on the Finsler structure, then for a region $A \subset \mathbf{R}^{n}$ with a Finsler structure $\Phi$ one has

$$
\operatorname{Vol}(A, \Phi)=\int_{A} \operatorname{Vol}\left(I^{n}, \Phi_{x}\right) d x
$$

where $I^{n}=[0,1]^{n}$ is the unit cube in $\mathbf{R}^{n}$, and $\Phi_{x}$ is a Banach norm in $\mathbf{R}^{n}$ (regarded as a flat Finsler structure) obtained from $\left.\Phi\right|_{T_{x} \mathbf{R}^{n}}$ via the identification $T_{x} \mathbf{R}^{n}=\mathbf{R}^{n}$.

For Busemann and symplectic volumes, this can be written as follows. For a point $x$ in a Finsler manifold $(M, \Phi)$, define $B_{x}=\left\{v \in T_{x} M: \Phi(v) \leq 1\right\}$ and $B_{x}^{*}=\left\{w \in T_{x}^{*} M: \Phi^{*}(w) \leq 1\right\}$. If $M=\mathbf{R}^{n}$, one identifies $T_{x} M, T_{x}^{*} M$ and $\mathbf{R}^{n}$, and then the formula (1) for Busemann volume takes the form

$$
\operatorname{Vol}(A, \Phi)=\frac{1}{\varepsilon_{n}} \int_{A} m_{n}\left(B_{x}\right)^{-1} d x
$$

and for symplectic volume one gets

$$
\operatorname{Vol}(A, \Phi)=\frac{1}{\varepsilon_{n}} \int_{A} m_{n}\left(B_{x}^{*}\right) d x
$$

where $m_{n}$ is the standard Eucludean volume in $\mathbf{R}^{n}$ and $\varepsilon_{n}$ is the volume of the $n$-dimensional Eucildean unit ball. 
Let Vol is a Finsler volume functional, $(M, \Phi)$ a Finsler manifold, $N$ a smooth manifold and $f: N \rightarrow M$ an immersion. We denote by $\operatorname{Vol}(f)$ or $\operatorname{Vol}(f, \Phi)$ the volume of $N$ with respect to the induced Finsler structure $f^{*} \Phi=\Phi \circ d f$.

This is generalized to arbitrary smooth and even Lipschitz maps (also known as "singular surfaces" in $M$ ) as follows. Let $f: N \rightarrow M$ is a Lipshitz map, then $f$ is differentiable almost everywhere and so yields a measurable pull-back "Finsler structure" $f^{*} \Phi=\Phi \circ d f$. Then one defines $\operatorname{Vol}(f)$ by integrating over $N$ the Finsler volume form determined by $f^{*} \Phi$. (The volume form is defined similarly to (1) at points $x \in N$ where $d_{x} f$ is injective, and equals zero at other points).

By additivity, this definition of volume extends to (Lipschitz) singular chains in Finsler manifolds, in particular, in finite-dimensional Banach spaces.

Note that a Lipschitz map can be approximated by smooth maps so that the derivatives converge in $L^{1}$ (implying that the volumes converge). This is what we will mean by saying that something "follows for Lipschitz surfaces by approximation".

2.3. Stable norms of periodic metrics. Let $(M, \Phi)$ be a Finsler manifold acted upon by $\mathbf{Z}^{n}$. For $z \in \mathbf{Z}^{n}$ and $x \in M$, the action of $z$ on $x$ is denoted by $x+z$. We assume that this action is co-compact, proper and by isometries. This means that $M / \mathbf{Z}^{n}$ is a compact Finsler manifold and the projection $M \rightarrow M / \mathbf{Z}^{n}$ is a covering map; moreover $\mathbf{Z}^{n}$ acts by deck transformations. We will call $(M, \Phi)$ a periodic Finsler manifold. As the leading example, one may think of the universal cover of a Finsler $n$-torus together with its deck group. In this case $M$ can be identified with $\mathbf{R}^{n}$ carrying a Finsler metric, which is invariant under translations by vectors from $\mathbf{Z}^{n}$.

The stable norm of $(M, \Phi)$ is a norm $\|\cdot\|$ on $\mathbf{R}^{n}$ defined as follows: fix an $x \in M$, then for a $z \in \mathbf{Z}^{n}$ set

$$
\|z\|=\lim _{k \rightarrow \infty} \frac{d_{\Phi}(x, x+k z)}{k} .
$$

It is easy to see that this limit exists, does not depend on $x$, and that the function $\|\cdot\|$ defined by this formula extends to a vector-space norm on $\mathbf{R}^{n}$.

Another way of defining the stable norm is the following

Bounded Distance Theorem ([Bu1]). Let $(M, \Phi)$ be a periodic Finsler manifold (more generally, a periodic length space) and $\|\cdot\|$ its stable norm. Then there exists a constant $C$ such that, for every $z \in Z^{n}$ and $x \in M$,

$$
\|z\| \leq d_{\Phi}(x, x+z) \leq\|z\|+C .
$$

In particular, this inequality implies that the Gromov-Hausdorff distance between $\left(M, d_{\Phi}\right)$ and the Banach space $\left(\mathbf{R}^{n}, d_{\|\cdot\|}\right)$ is finite. Note that this property defines the Banach space $\left(\mathbf{R}^{n}, d_{\|\cdot\|}\right)$ up to a (linear) isometry. Thus 
the isometry class of $\left(\mathbf{R}^{n}, d_{\|\cdot\|}\right)$ remains the same if one chooses another action of $\mathbf{Z}^{n}$ on $(M, \Phi)$.

As the matter of fact, our definition is slightly different from the classical way of defining the stable norm on $H_{1}\left(M / \mathbf{Z}^{n}, \mathbf{R}\right)$. They coincide, however, in the case of the universal abelian cover; in general situation, $\|\cdot\|$ arises as the projection of the norm from $H_{1}\left(M / \mathbf{Z}^{n}, \mathbf{R}\right)$.

\section{Conjectures. Statement of the Results}

3.1. Main conjectures and their equivalence. In this section, a particular choice of volume functional Vol does not play any role. This may be symplectic volume, Hausdorff measure, or any other measure monotone with respect to $\Phi$.

We begin with the following definition. Let $\left(M^{n}, \Phi\right)$ be a periodic Finsler manifold. The asymptotic volume growth $\nu(M, \Phi)$ is defined by

$$
\nu(M, \Phi)=\lim _{r \rightarrow \infty} \frac{\operatorname{Vol}\left(B_{r}(x)\right)}{r^{n}},
$$

where $B_{r}(x)$ is the metric ball of a radius $r$ centered at a point $x \in M$.

This limit exists and does not depend on $x$, moreover $\nu(M, \Phi)$ can be expressed by the following formula:

$$
\nu(M, \Phi)=\operatorname{Vol}\left(M / Z^{n}\right) \cdot m_{n}(B)
$$

where $B \subset \mathbf{R}^{n}$ is the unit ball of the stable norm of $(M, \Phi)$, and $m_{n}$ is the Lebesgue measure in $\mathbf{R}^{n}$. Indeed, the Bounded Distance Theorem implies that the ball $B_{r}(x)$ in $(M, \Phi)$ covers the quotient space $M / \mathbf{Z}^{n}$ appoximately $N(r)$ times, where $N(r)=\operatorname{card}\left(r B \cap \mathbf{Z}^{n}\right)$, that is the number of integral points in the the $r$-ball of the stable norm. Hence $\operatorname{Vol}\left(B_{r}(x)\right) \sim$ $N(r) \cdot \operatorname{Vol}\left(M / \mathbf{Z}^{n}\right)$. Obviously $N(r)$ asymptotically equals $r^{n} \cdot m_{n}(B)$, so the formula (4) follows.

We are now in a position to state our main conjectures. First we formulate them in the simplest topological set-up.

Conjecture A. Let $(M, \Phi)$ be the universal cover of a Finsler n-torus (in other words, $\Phi$ is a Finsler metric on $M=\mathbf{R}^{n}$ invariant under $\mathbf{Z}^{n}$ acting by translations). Then $\nu(M, \Phi) \geq \nu\left(\mathbf{R}^{n},\|\cdot\|\right)$, where $\|\cdot\|$ is the stable norm of $(M, \Phi)$.

Conjecture B. Let $X$ be a finite-dimensional Banach space, $V \subset X$ be an $n$-dimensional affine subspace, $A \subset V$ be a region diffeomorphic to the disc $D^{n}$, and $f: D^{n} \rightarrow X$ be an immersion (more generally, a Lipschitz map) such that $\left.f\right|_{\partial D^{n}}$ is a diffeomorphism onto $\partial A$. Then $\operatorname{Vol}(f) \geq \operatorname{Vol}(A)$.

Note that the Lipschitz version of this conjecture follows by approximation from the one with immersions (assuming that the latter holds for spaces $X$ of any dimension). Indeed, one can approximate any Lipschitz map from $D^{n}$ to $X$ by an immersion to a higher-dimensional space $X^{\prime} \supset X$. 
Conjecture C. Let $A$ be a bounded region in an $n$-dimensional Banach space $\left(V^{n},\|\cdot\|\right)$. Let $\Phi$ be a Finsler metric on A such that

$$
d_{\Phi}(x, y) \geq\|x-y\|
$$

for all $x, y \in \partial A$. Then $\operatorname{Vol}(A, \Phi) \geq \operatorname{Vol}(A,\|\cdot\|)$.

Theorem 1. For every $n$, the three conjectures $A, B$ and $C$ are equivalent.

3.2. Minimality and ellipticity. ¿From now on, we will be concerned with symplectic volume. We reserve the notation Vol for this volume. If we want to stress a particular dimension, we can write $n$-volume or $\mathrm{Vol}_{n}$; for instance, 2-volume is symplectic surface area.

One may notice that Conjecture B naturally embeds into a classical scheme. Namely, the Finsler volume $\mathrm{Vol}_{n}$ defines an area functional on $n$-dimensional surfaces in a Banach space $X$ (more generally, on Lipschitz singular chains in $X)$. We fix an $(n-1)$-dimensional chain $\gamma=\partial A$ contained in an $n$-dimensional affine subspace of $X$ and want to minimize $\operatorname{Vol}(\beta)$ subject to $\partial \beta=\gamma$. One then asks if a minimum is attained on a chain contained in the affine subspace. In Conjecture 2, we required that $\beta$ is represented by a topological disc. Thus, one formulates the following "homological" version of Conjecture B:

Conjecture B'. Let $\alpha$ be a topological $n$-disc in an n-dimensional affine subspace of a Banach space $X$. Then $\alpha$ has minimal volume among all $n$ dimensional singular chains $\beta$ with integral coefficients such that $\partial \beta=\partial \alpha$.

This conjecture is equivalent to that the $n$-dimensional volume functional is elliptic (over $\mathbf{Z}$ ) in any Banach space whose norm is quadratically convex. Ellipticity is a classical and very important property in the theory of minimal surfaces. We will use the term ellipticity for the property formulated in Conjecture B', even if the Banach norm is not quadratically convex. Conjecture B' (as well as Conjecture B) has been first formulated by Busemann (see, for instance, [BES] and refTh), who also proved it for codimension 1 (that is, when the dimension of the Banach space is $n+1$ ).

Conjecture B' obviously implies Conjecture B. In fact, the two conjectures are equivalent for every $n \geq 3$. Indeed, for $n \geq 3$ any $n$-chain over $\mathbf{Z}$ whose boundary is a topological sphere can be replaced by an immersed $n$-disc spanning the same boundary and having almost the same volume (see [Gr], Appedix 2, Proposition A'). In dimension $n=2$, one has to consider not only immersed discs but also discs with handles.

The assumption that we are looking for a chain with integral coefficients is essential. The following theorem presents a counter-example to a version of this conjecture over rational coefficients. It also leaves little hope to have a "simple" argument poving the conjecture. At least, classical methods of proving ellipticity do not distinguish integer versus real coefficients.

Theorem 2. There exist a four-dimensional Banach space $X$, a two-dimensional affine subspace $V \subset X$, a disc $\alpha \subset V$, and a chain $\beta$ in $X$ represented by an 
immersed disc such that $\partial \beta=N \cdot \partial \alpha$ and $\operatorname{Vol}_{2}(\beta)<N \operatorname{Vol}_{2}(\alpha)$, for some natural number $N$ (in fact, one can take $N=10$ ).

On the positive side, the following theorem holds:

Theorem 3. Conjectures $A, B, C$ are true for dimension $n=2$.

Therefore, Conjecture $\mathrm{B}^{\prime}$ ' is true in dimension 2 for chains $\beta$ represented by immersed discs (versus "discs with handles").

There are some non-trivial situations when ellipticity can be shown, namely there are spaces whose 2-volume functional is elliptic over intergers but it fails to be elliptic over reals or rationals:

Theorem 4. There exist a four-dimensional Banach space $X$ and a twodimensional affine subspace $V \subset X$ such that a disc $\alpha \subset V$ minimizes area among the chains with integral coefficients spanning the same boundary $\partial \alpha$, but does not minimize area among the chains with rational coefficients spanning $\partial \alpha$.

Proof of this theorem will be published elsewhere.

Let us also mention that Conjecture B' is true in dimension 2 for Lipschits chains whose tangent is sufficiently close to $\alpha$ (a.e.), and hence 2 -dimensional affine planes in normed spaces are (at least) locally area minimizers (in the strongest sense):

Lemma 3.1. Let $\gamma=\partial \alpha$, where $\alpha$ is a topological $n$-dimensional disc in an $n$-dimensional affine subspace of a Banach space $X$. There exists a positive $\varepsilon$ such that a minimum of $\operatorname{Vol}(\beta)$ subject to $\partial \beta=\gamma$ over all smooth chains $\beta$ over $Q$ whose tangent space is $\varepsilon$-close to $\alpha$ a.e. is attained at $\alpha$.

A proof of this Lemma can be found in Section 6.1.

3.3. Gaussian measures of surfaces. The reason why volume functional might fail to enjoy ellipticity can be seen by looking at volume form on the Grassmanian cone in the space of $n$-vectors. It has been noticed by Busemann that volume form may fail to be convex (i.e. extendable to a convex function on all $n$-vectors). Namely, there exists a four-dimensional Banach space $X$ and three simple 2-vectors $a, b$ and $c$ whose sum $a+b+c$ is also a simple 2 -vector and

$$
\operatorname{Vol}(a)+\operatorname{Vol}(b)+\operatorname{Vol}(c)<\operatorname{Vol}(a+b+c)
$$

(where $\operatorname{Vol}(e)$ might be any of the Busemann and the Holmes-Thompson areas of a parallelogram spanned by $e$ ). This means that norms induced by Busemann and Holmes-Thompson areas on the set of simple 2-vectors in $X$ cannot be extended to a convex function on the space $X \wedge X$ of all 2-vectors (see $[\mathrm{BES}])$

If one could construct a polyhedron whose boundary belongs to an affine plane and whose faces represent the 2 -vectors $a, b$ and $c$, this would immediately give a counter-example to the Conjectures. This suggests the following 
problem in classical Euclidean geometry. For simplicity, we discuss it for two dimensional surfaces.

Let us begin with an oriented polyhedral surface in $\mathbf{R}^{m}$. Each (oriented) face of the surface represents a 2-vector (which is parallel to the face and whose length is equal to the Euclidean area of the face). If the polyhedron has parallel faces, we add the corresponding 2-vectors together. This way we obtain a finite collection of simple 2-vectors, which will be called the Gaussian measure. Equivalently, for each face of the polyhedral surface, one introduces an atomic measure on the Grassmanian manifold of oriented 2-planes. This measure is concentrated at the point representing the face and its weight is equal to the Euclidean 2-volume of the face. The sum of these measures gives nothing but the same weighted directions of faces.

More generally, for a smooth surface, the push-forward of Euclidean 2volume under the Gaussian map defines a measure on the Grassmanian manifold. This measure will aslo be called the Gaussian measure of the surface.

We are concerned with the following problem, which can be asked for both polyhedral and smooth surfaces:

Given an (atomic) measure on a Grassmanian manifold, we want to know whether it can be approximated (with any given precision) by the Gaussian measure of a (polyhedral) surface whose boundary is a simple curve in a 2-dimensional affine subspace $V$.

A more natural question (which is, however, not quite equivalent to this one) is which measures on the Grassmannian can be appoximated (with any given precision) by a directional measure of a closed surface.

To illustrate why we want to approximate a measure instead of trying to get it precisely, let us consider the following example regarding 2-dimensional surfaces in $\mathbf{R}^{3}$.

Let us choose three vectors $a, b, c \in \mathbf{R}^{3}$ with $a+b+c=0$, and a vector $d$, and consider the atomic measure concentaited on $a \wedge d, b \wedge d$ and $c \wedge d$ with equal weights on each. Geometrically, the faces of a polyhedral surface with this Gaussian image are parallel to three planes that have a line in common. It is easy to see that such surface cannot be closed. However, adding an arbitrarily small amount of faces parallel to any direction transversal to $d$ allows one to construct a closed surface (which looks like a long prizm with two small lids) with this Gaussian measure.

There is an obvious linear constraint. To a point in the Grassmannian manifold, one associates a unit 2-vector. Integrating this function against the directional measure of a surface whose boundary belongs to $V$, one obviously gets a simple 2-vector parallel to $V$. Analogously, for a closed surface this integration must yield the zero 2-vector (a well-known physical interpretation is the fact that the total force produced by the air pressure is zero).

It is not difficult to show that, in co-dimension one, this (linear relation) is the only constraint. The reason is that every $(m-1)$-vector in $\mathbf{R}^{m}$ is 
simple. Polyheral surfaces with approximately given directional measure can be constructed by an inductive procedure. Loosely speaking, each step of the induction consists in merging two atomic weights in the directional measure similarly to the example with a prizm.

In higher co-dimesions, there are other constraints, whose nature remains rather obscure. The fact that Conjecture B is true for two-dimensional surfaces already implies the existance of such constraints for surfaces represented by immersed discs (otherwise, a counterexample could be constructed out of Busemann's example of four two-vectors with a "reversed triangle inequality" at the beginning of this section). Moreover, the proof of Theorem 4 is based on extracting a (rather clumsy) non-linear constraint of this sort.

3.4. A related problem in convex geometry. For the reader who does not want to think about Banach spaces, we can suggest a rather classical formulation of a problem in Euclidean geometry, which seems to incorporate many of the features of Conjecture B. On the other hand, this problem does not have its analogs in intrinsic geometry that would be counterparts of Conjectures A and C, and this circumstance leaves less diversity in possible approaches to this problem (note that our proof for dimension two is essentially intrinsic). The problem reads as follows:

Consider a (metric) $n$-1-sphere $\alpha$ and a convex body $B$ in an $m$ dimensional Euclidean space $(m>n)$. Is it true that a minimum of the Euclidean volume of the Minkowski sum $\beta+B$ over all surfaces with boundary $\partial \beta=\alpha$ is attained on the (metric) disc bounded by $\alpha$ ?

The answer is "yes" for surfaces of co-dimension 1, that is for $m=n+1$, and also for $n=1$ (the proof we know is an easy argument relying on the Stokes' Theorem). In the latter case, the sphere is just a pair of points, "surfaces" are curves connecting the points, and the problem basically asks whether, given two points, a straight tunnel has the smallest volume among all tunnels between that are enough to carry $B$ (without tilting it) from one point to the other.

\section{Equivalence of Conjectures}

In this section we prove Theorem 1, namely that the conjectures A (about the asymptotic volume growth), B (about minimality of flats) and C (about filling volume) are equivalent for any given dimension $n$.

We begin with simple implications $\mathrm{A} \Longrightarrow \mathrm{C} \Longrightarrow \mathrm{B}$.

4.1. Conjecture $\mathbf{C} \Longrightarrow$ Conjecture B. Let $A \subset V \subset X$ be as in Conjecture $\mathrm{B}$, and let $\|\cdot\|$ denote the Banach norm of $X$. It suffices to prove that $\operatorname{Vol}(f) \geq \operatorname{Vol}(A)$ for any immersion $f: A \rightarrow X$ such that $\left.f\right|_{\partial A}$ is the identity map. Let $\Phi$ denote the Finsler metric on $A$ induced by $f$. Then $d_{\Phi}(x, y) \geq\|x-y\|$ for all $x, y \in \partial A$. Indeed, $d_{\Phi}(x, y)$ is the infimum of lengths of curves connecting $x$ and $y$ in the surface parameterized by $f$, and the length of any curve in $(X,\|\cdot\|)$ connecting $x$ and $y$ is no less than 
$\|x-y\|$. Applying Conjecture $\mathrm{C}$ to $(V,\|\cdot\|), A$ and $\Phi$, we obtain that $\operatorname{Vol}(f)=\operatorname{Vol}(A, \Phi) \geq \operatorname{Vol}(A,\|\cdot\|)$.

4.2. Conjecture $\mathbf{A} \Longrightarrow$ Conjecture C. Let $(V,\|\cdot\|), A$ and $\Phi$ be as in Conjecture C. Introduce a lattice $\Gamma \simeq \mathbf{Z}^{n}$ in $V$ such that the images of $A$ under translations by elements of $\Gamma$ are disjoint. Then define a Finsler structure $\Phi^{\prime}$ on $V$ as follows: $\Phi^{\prime}$ is the parallel translation of $\Phi$ in every region $A+z$ where $z \in \Gamma$, and $\Phi^{\prime}=\|\cdot\|$ outside $A+\Gamma$. Then $\Phi^{\prime}$ is $\Gamma$ periodic and piecewise continuous. Let $\|\cdot\|^{\prime}$ denote the stable norm of $\Phi^{\prime}$. The assumption that $d_{\Phi} \geq\|\cdot\|$ on $\partial A$ implies that $d_{\Phi^{\prime}} \geq\|\cdot\|$ everywhere and hence $\|\cdot\|^{\prime} \geq\|\cdot\|$. Then by Conjecture A (extended to piecewise continuous Finsler structures by approximation) one has $\nu\left(V, \Phi^{\prime}\right) \geq \nu\left(V,\|\cdot\|^{\prime}\right)$. Since

$$
\frac{\nu\left(V, \Phi^{\prime}\right)}{\operatorname{Vol}\left(V / \Gamma, \Phi^{\prime}\right)}=\frac{\nu\left(V,\|\cdot\|^{\prime}\right)}{\operatorname{Vol}\left(V / \Gamma,\|\cdot\|^{\prime}\right)}
$$

by (4), it follows that

$$
\operatorname{Vol}\left(V / \Gamma, \Phi^{\prime}\right) \geq \operatorname{Vol}\left(V / \Gamma,\|\cdot\|^{\prime}\right) \geq \operatorname{Vol}(V / \Gamma,\|\cdot\|) .
$$

Then $\operatorname{Vol}(A, \Phi)=\operatorname{Vol}\left(A, \Phi^{\prime}\right) \geq \operatorname{Vol}(A,\|\cdot\|)$ because $\Phi^{\prime}$ and $\|\cdot\|$ coincide outside $A+\Gamma$.

4.3. Calibrating functions. In the rest of this section we prove the implication Conjecture $\mathrm{B} \Longrightarrow$ Conjecture A (i. e., we prove the volume growth inequality assuming minimality of flats in Banach spaces). We begin with several constructions involving periodic metrics. (These constructions are also used in the next section).

Let $M$ be a manifold equipped with a $\mathbf{Z}^{n}$-periodic length metric $d$ and $\|\cdot\|$ its stable norm. This norm is defined on the vector space $V \simeq \mathbf{Z}^{n} \otimes \mathbf{R}=\mathbf{R}^{n}$. We denote by $V^{*}$ the space of linear functions from $V$ to $\mathbf{R}$ and by $\|\cdot\|^{*}$ the norm dual to $\|\cdot\|$, that is the norm on $V^{*}$ defined by

$$
\|h\|^{*}=\sup \{h(x):\|x\| \leq 1\} .
$$

Let $B^{*}$ denote the unit ball of $\|\cdot\|^{*}$. Its boundary $\partial B^{*}$ is the unit sphere of $\|\cdot\|^{*}$, i.e. the set of unit-norm linear functions.

Definition 4.1. We say that a function $F: M \rightarrow \mathbf{R}$ calibrates a linear function $h \in V^{*}$ if

(1) $F$ is a Lipschitz-1 function, i.e. $|F(x)-F(y)| \leq d(x, y)$ for all $x, y \in$ $M$.

(2) $F(x+z)=F(x)+h(z)$ for all $x \in M, z \in \mathbf{Z}^{n}$.

Remark 4.2. If $F$ satisfies the above definition, its derivative $d F$ projects down to $M / \mathbf{Z}^{n}$ as a (measurable) closed 1-form which calibrates the length functional and whose de Rham cohomology class (projected to $V^{*}$ ) equals $h$. It is known that the definition of the stable norm in terms of such 1-forms can be interpreted as follows: $\|h\|^{*}=\inf \{c>0$ : there exists a function calibrating $h / c\}$. 
Proposition 4.3. For every $h \in \partial B^{*}$ there exist a function $F: M \rightarrow \mathbf{R}$ calibrating $h$ and satisfying an additional property: for every $x \in M$ there is a unit-speed geodesic ray $\gamma:[0,+\infty) \rightarrow M$ such that $\gamma(0)=x$ and $F(\gamma(t))=F(x)+t$ for all $t$.

Proof. Fix a point $x_{0} \in M$ and define

$$
F(x)=\limsup _{z \in \mathbf{Z}^{n},|z| \rightarrow \infty}\left(h(z)-d\left(x, x_{0}+z\right)\right)
$$

for an $x \in M$. We will prove that $F$ is a desired calibrating function.

1. The values of $F$ are finite. Indeed, since $\|h\|^{*}=1$, one has $|h(z)| \leq\|z\|$ for all $z \in \mathbf{Z}^{n}$ and there is a sequence $\left\{z_{i}\right\}_{i=1}^{\infty}$ in $\mathbf{Z}^{n}$ such that $\left|z_{i}\right| \rightarrow \infty$ and $\left\|z_{i}\right\|-h\left(z_{i}\right) \leq$ const. By the Bounded Distance Theorem,

$$
\|z\| \leq d\left(x_{0}, x_{0}+z\right) \leq\|z\|+\text { const }
$$

for all $z \in \mathbf{Z}^{n}$. Hence

$$
h(z)-d\left(x, x_{0}+z\right) \leq h(z)-d\left(x_{0}, x_{0}+z\right)+d\left(x_{0}, x\right) \leq d\left(x_{0}, x\right)
$$

for all $z$, and

$$
h\left(z_{i}\right)-d\left(x, x_{0}+z_{i}\right) \geq h(z)-d\left(x_{0}, x_{0}+z\right)-d\left(x_{0}, x\right) \geq-d\left(x_{0}, x\right)-\text { const }
$$

for all $i$. It follows that $-d\left(x_{0}, x\right)-$ const $\leq F(x) \leq d\left(x_{0}, x\right)$.

2. $F$ is Lipschitz-1 as an upper limit of Lipschitz-1 functions.

3. The second condition from Definition 4.1 follows from $Z^{n}$-periodicity of the metric:

$$
\begin{aligned}
F(x+z) & =\limsup _{z^{\prime} \in \mathbf{Z}^{n},\left|z^{\prime}\right| \rightarrow \infty}\left(h\left(z^{\prime}\right)-d\left(x+z, x_{0}+z^{\prime}\right)\right) \\
& =\limsup _{z^{\prime} \in \mathbf{Z}^{n},\left|z^{\prime}\right| \rightarrow \infty}\left(h\left(z^{\prime}+z\right)-d\left(x+z, x_{0}+z^{\prime}+z\right)\right) \\
& =\limsup _{z^{\prime} \in \mathbf{Z}^{n},\left|z^{\prime}\right| \rightarrow \infty}\left(h\left(z^{\prime}\right)-d\left(x, x_{0}+z^{\prime}\right)\right)+h(z)=F(x)+h(z)
\end{aligned}
$$

(the second equality is obtained by a change of variables $z^{\prime} \mapsto z^{\prime}+z$ ).

4. Given $x \in M$, there exists a sequence $\left\{z_{i}\right\}_{i=1}^{\infty}$ in $\mathbf{Z}^{n}$ such that $\left|z_{i}\right| \rightarrow \infty$ and $F(x)=\lim _{i \rightarrow \infty}\left(h\left(z_{i}\right)-d\left(x, x_{0}+z_{i}\right)\right)$. Let $\gamma_{i}:\left[0, d\left(x, x_{0}+z_{i}\right)\right] \rightarrow M$ be a minimal geodesic joining $x$ and $x_{0}+z_{i}$. A standard compactness argument shows that a subsequence of $\left\{\gamma_{i}\right\}$ converges pointwise to a geodesic ray $\gamma:[0,+\infty) \rightarrow M$. Then $\gamma$ satifies $F(\gamma(x+t))=F(x)+t$ for all $t$. Indeed,

$$
\begin{aligned}
F(\gamma(t)) & \geq \limsup _{i \rightarrow \infty}\left(h\left(z_{i}\right)-d\left(\gamma(t), x_{0}+z_{i}\right)\right) \\
& =\limsup _{i \rightarrow \infty}\left(h\left(z_{i}\right)-d\left(\gamma_{i}(t), x_{0}+z_{i}\right)\right) \\
& =\limsup _{i \rightarrow \infty}\left(h\left(z_{i}\right)-d\left(x, x_{0}+z_{i}\right)\right)+t=F(x)+t
\end{aligned}
$$

The inverse inequality $F(\gamma(t)) \leq F(x)+t$ follows from the fact that $F$ is a Lipschitz-1 function. The proposition follows. 
Remark 4.4. The formula for $F$ in the above proof is similar to the classical definition of Busemann functions. The Busemann function of a geodesic ray $\gamma$ is obtained from distance functions of points $\gamma(t)$ as $t \rightarrow \infty$. The above formula for $F$ can be interpreted as the same construction applied to distance functions of a family of parallel "hyperplanes" $h^{-1}(t)$ in $\mathbf{Z}^{n} \subset M$, where the inclusion $\mathbf{Z}^{n} \hookrightarrow M$ is given by $z \mapsto x_{0}+z$. (In fact, one could use affine hyperplanes if $M$ is the universal cover of an $n$-torus and is identified with $\mathbf{R}^{n}$.) In this interpretation, the geodesic constructed in the last part of the proof is an analog of an asymptotic ray.

4.4. Equivariant embeddings. Consider the vector space $\ell_{\infty}\left(\partial B^{*}\right)$ of bounded real-valued funtions on $\partial B^{*}$ equipped with the standard norm $\|\cdot\|_{\infty},\|f\|_{\infty}=$ $\sup |f|$. Then $V$ admits a canonical linear embedding $I$ into $\ell_{\infty}\left(\partial B^{*}\right)$, namely the image $I(v)$ of a $v \in V$ is the function given by $I(v)(h)=h(v)$. The duality theorem for convex bodies (saying that $B^{* *}=B$ ) implies that $I$ is an isometric map, i.e. $\|I(v)\|_{\infty}=\|v\|$ for all $v \in V$.

Calibrating functions discussed in the previous section allow us to contruct a Lipschitz-1 equivariant map $I_{M}: M \rightarrow \ell_{\infty}\left(\partial B^{*}\right)$, where equivariance is considered with respect to the action of $\mathbf{Z}^{n}$ in $M$ and the action of $I\left(\mathbf{Z}^{n}\right)$ in $\ell_{\infty}\left(\partial B^{*}\right)$ by parallel translations, namely that $I_{M}(x+z)=I_{M}(x)+I(z)$ for all $x \in M, z \in \mathbf{Z}^{n}$. Since $I_{M}$ is Lipschitz and equivariant, its image stays within a bounded distance from the linear $n$-subspace $I(V) \subset \ell_{\infty}\left(\partial B^{*}\right)$.

Such a map $I_{M}$ can be constructed as follows. Fix a point $x_{0} \in M$ and associate to every $h \in \partial B^{*}$ a calibrating function $F_{h}$ such that $F_{h}\left(x_{0}\right)=0$ (this can be achived because one may add constants to calibrating functions). Define $I_{M}(x)(h)=F_{h}(x)$ for $x \in M, h \in \partial B^{*}$. Then $I_{M}(x)$ is a bounded function on $\partial B^{*}$, so $I_{M}$ is a map from $M$ to $\ell_{\infty}\left(\partial B^{*}\right)$. The Lipschitz-1 property and equivariance follow immediately from the two conditions of Definition 4.1.

In fact, we will use only finite-dimensional approximations to these maps. Let $\mathbf{R}_{\infty}^{N}$ denote the space $\mathbf{R}^{N}$ equipped with the $l_{\infty}$-norm $\|\cdot\|_{\infty}$ given by $\left\|\left(x_{1}, \ldots, x_{N}\right)\right\|_{\infty}=\max \left|x_{i}\right|$. Given a collection $h_{1}, \ldots, h_{N} \in \partial B^{*}$, one defines a coordinate projection $P_{h_{1}, \ldots, h_{N}}: \ell_{\infty}\left(\partial B^{*}\right) \rightarrow \mathbf{R}_{\infty}^{N}$ by $P_{h_{1}, \ldots, h_{N}}(f)=$ $\left(f\left(h_{1}\right), \ldots, f\left(h_{N}\right)\right)$. This projection is obviously a Lipschitz-1 map. Composing $P_{h_{1}, \ldots, h_{N}}$ with $I$ and $I_{M}$, one obtains Lipschitz-1 maps

and

$$
I_{h_{1}, \ldots, h_{N}}: V \rightarrow \mathbf{R}_{\infty}^{N}, \quad x \mapsto\left(h_{1}(x), \ldots, h_{N}(x)\right)
$$

$$
I_{M, h_{1}, \ldots, h_{N}}: M \rightarrow \mathbf{R}_{\infty}^{N}, \quad x \mapsto\left(F_{h_{1}}(x), \ldots, F_{h_{N}}(x)\right) .
$$

If $\left\{h_{1}, \ldots, h_{N}\right\}$ is a sufficiently fine net in $\partial B^{*}$, the map $I_{h_{1}, \ldots, h_{N}}$ is "almost isometric" in the Lipschitz sense, i.e.

$$
(1-\varepsilon)\|v\| \leq\left\|I_{h_{1}, \ldots, h_{N}}(v)\right\|_{\infty} \leq\|v\|
$$

for all $v$, where $\varepsilon$ can be made arbitrarily small by the choice of $N$ and $\left\{h_{i}\right\}$. Indeed, the pull-back of $\|\cdot\|_{\infty}$ under $I_{h_{1}, \ldots, h_{N}}$ is the norm on $V$ whose unit ball is the polyhedron $\left\{v \in V:\left|h_{i}(v)\right| \stackrel{\leq}{\leq} 1\right.$ for all $\left.v\right\}$. Such a polyhedron 
(i.e. a polyhedron bounded by a collection of hyperplanes supporting $B$ ) can approximate $B$ arbitrarily close, and the above inequality holds whenever it is contained in the set $(1-\varepsilon)^{-1} B$.

4.5. Conjecture $\mathbf{B} \Longrightarrow$ Conjecture A. Now assume that $(M, \Phi)$ is the universal cover of a Finsler $n$-torus and $d=d_{\Phi}$. We identify $M$ with $\mathbf{R}^{n}$, so $\Phi$ is a $\mathbf{Z}^{n}$-periodic Finsler structure in $\mathbf{R}^{n}$ and the stable norm $\|\cdot\|$ is defined on $V=\mathbf{R}^{n}$.

Fix a small $\varepsilon>0$ and choose a collection $h_{1}, \ldots, h_{N} \in \partial B^{*}$ such that

$$
\left\|I_{h_{1}, \ldots, h_{N}}(v)\right\|_{\infty} \geq(1-\varepsilon)\|v\|
$$

for all $v \in V$ (see the previous section). For brevity, we denote the maps $I_{h_{1}, \ldots, h_{N}}: V \rightarrow \mathbf{R}_{\infty}^{N}$ and $I_{M, h_{1}, \ldots, h_{N}}: M \rightarrow \mathbf{R}_{\infty}^{N}$ by $I$ and $I_{M}$ resp. Since $I_{M}$ is a Lipschitz-1 map, it is a volume non-increasing map.

Given an $r>0$, consider a surface $F$ in $\mathbf{R}_{\infty}^{N}$ parameterized by the map $I_{M}$ restricted to $r B \subset V=M$ (note that $r B$ is the ball of radius $r$ in the space $(V,\|\cdot\|))$. Consider a surface $F_{c}$ in $\mathbf{R}_{\infty}^{N}$ parameterized by $\partial(r B) \times[0,1]$ so that every fiber $x \times[0,1]$ (where $x \in \partial(r B)$ ) parametrizes the straight line segment from $I_{M}(x)$ to $I(x)$. This surface is a "collar" joining the boundaries $I_{M}(\partial(r B))$ and $I(\partial(r B))$ in $\mathbf{R}_{\infty}^{N}$. Since $I$ and $I_{M}$ are Lipschitz maps and $\left\|I_{M}(x)-I(x)\right\|_{\infty}$ is bounded uniformly in $x$, the volume of the collar has an upper bound of the form const $\cdot r^{n-1}$. The union of $F$ and $F_{c}$ is a Lipschitz surface (parameterized by a disc) spanning the boundary $I(\partial(r B))=\partial(I(r B))$. Since $I(r B) \subset I(V)$ and $I(V)$ is an $n$-dimensional linear subspace of $R_{\infty}^{N}$, Conjecture B yields that $\operatorname{Vol}(F)+\operatorname{Vol}\left(F_{c}\right) \geq \operatorname{Vol}(I(r B))$. Thus

$$
\begin{aligned}
\operatorname{Vol}(r B, \Phi) & \geq \operatorname{Vol}(F) \geq \operatorname{Vol}(I(r B))-\operatorname{Vol}\left(F_{c}\right) \\
& \geq(1-\varepsilon)^{n} \operatorname{Vol}(B,\|\cdot\|) r^{n}-\text { const } \cdot r^{n-1} .
\end{aligned}
$$

On the other hand, the Bounded Distance Theorem implies that there is a constant $C$ such that the set $r B$ is contained in the ball (with respect to the metric $d_{\Phi}$ ) of a radius $r+C$ centered at 0 . Therefore

$$
\begin{aligned}
\nu(M, \Phi) & \geq \lim _{r \rightarrow \infty} \frac{(1-\varepsilon)^{n} \operatorname{Vol}(B,\|\cdot\|) r^{n}-\text { const } \cdot r^{n-1}}{(r+C)^{n}} \\
& =(1-\varepsilon)^{n} \operatorname{Vol}(B,\|\cdot\|)=(1-\varepsilon)^{n} \cdot \nu(V,\|\cdot\|) .
\end{aligned}
$$

Since $\varepsilon$ is arbitrary, Conjecture A follows.

\section{TWO-DIMENSIONAL CASE}

The goal of this section is to prove Theorem 3 . We will do it by showing that the Volume Growth Conjecture $\mathrm{A}$ is true in dimension $n=2$. Let $(M, \Phi)$ be the universal cover of a Finsler 2-torus and $\|\cdot\|$ its stable norm defined on $V \cong \mathbf{R}^{2}$. We keep on using terms and notations for periodic metrics and stable norms introduced in the previous sections.

Since $\|\cdot\|$ and $\nu(M, \Phi)$ depend continuously on $\Phi$, we may assume that $\Phi$ is smooths and quadratically convex. 
We denote by $U T^{*} M$ the unit co-tangent bundle of $(M, \Phi)$, that is, $U T^{*} M=\{w \in T * M: \Phi(w) \leq 1\}$. Identify $M$ and $V$ by means of a $\mathbf{Z}^{2}$-equivariant diffeomorphism and fix an orientation on these spaces. This orientation induces a cyclic ordering on the unit sphere $\partial B^{*}$ in $V^{*}$ and the fibers of the unit co-tangent bundle $U T^{*} M$ (note that $\partial B^{*}$ and the fibers of $U T^{*} M$ are homeomorphic to $\left.S^{1}\right)$.

Lemma 5.1. Let functions $F_{1}, F_{2}, F_{3}: M \rightarrow \mathbf{R}$ calibrate distinct linear functions $h_{1}, h_{2}, h_{3} \in \partial B^{*}$ and satisfying the additional property from Proposition 4.3. Let $x \in M$ and suppose that derivatives $d_{x} F_{1}, d_{x} F_{2}$ and $d_{x} F_{3}$ exist. Then these derivatives are unit co-vectors and their cyclic order in $U T_{x}^{*} M$ is the same as that of $h_{1}, h_{2}$ and $h_{3}$ in $\partial B^{*}$.

Proof. For $i=1,2,3$ let $\gamma_{i}:[0,+\infty) \rightarrow M$ be a unit-speed geodesic ray such that $\gamma_{i}(0)=x$ and $F_{i}\left(\gamma_{i}(t)\right)=F_{i}(x)+t$ (cf. Proposition 4.3). Then $g_{i}^{\prime}(0)$ is a unit vector and $d_{x} F_{i}\left(g_{i}^{\prime}(0)\right)=1$, implying that $\left\|d_{x} F_{i}\right\| \geq 1$. On the other hand, $\left\|d_{x} F_{i}\right\| \leq 1$ because $F_{i}$ is a Lipschitz- 1 function. Hence $d_{x} F_{i} \in U T_{x}^{*} M$.

The stable norm of a two-dimensional torus (with smooth quadratically convex metric) is strictly convex in the sense that the boundary of its unit ball contains no straight line segments (cf. [Ba]). Hence for every $i=1,2,3$ there is a unique vector $v_{i} \in \partial B$ such that $h_{i}\left(v_{i}\right)=1$. Moreover $v_{i}$ and $h_{i}$ are related by an orientation-preserving homeomorphism from $\partial B$ to $\partial B^{*}$. A similar homeomorphism (in fact, the Legendre tranform) from $U T_{x} M$ to $U T_{x}^{*} M$ maps the derivatives $\left\|d_{x} F_{i}\right\|$ to vectors $\gamma_{i}^{\prime}(0)$. Thus it suffices to prove that the vectors $\gamma_{i}^{\prime}(0)$ in $U T_{x} M$ are cyclically ordered in the same way as the $v_{i}$ in $\partial B$.

Since we have identified $M$ and $V$, we have a (non-invariant) vector space structure on $M$. Using this, the bounded distance theorem can be wriiten as

Hence

$$
\left|d_{\Phi}(y, z)-\|y-z\|\right| \leq \text { const }, \quad x, y \in M
$$

$$
\limsup _{t \rightarrow \infty} \frac{\left\|\gamma_{i}(t)-x\right\|}{t} \leq 1
$$

because $\gamma_{i}$ is a unit-speed geodesic. On the other hand, the function $F_{i}-h_{i}$ is $\mathbf{Z}^{2}$-periodic and hence bounded. By the choice of $\gamma_{i}$ we then have

$$
\lim _{t \rightarrow \infty} \frac{h_{i}\left(\gamma_{i}(t)-x\right)}{t}=\lim _{t \rightarrow \infty} \frac{F_{i}\left(\gamma_{i}(t)\right)-F_{i}(x)}{t}=1 .
$$

It follows that $\frac{\gamma_{i}(t)-x}{t}$ converges to $v_{i}$ as $t \rightarrow \infty$.

For a $T>0$ let $p_{i}(T)$ denote the first intersection point of $\gamma_{i}$ and the convex Jordan curve $\Gamma_{T}=x+T \cdot \partial B \subset V$. Then $p_{i}(T) / T \rightarrow v_{i}$ as $T \rightarrow \infty$. Thus if $T$ is large enough, the points $p_{1}(T), p_{2}(T)$ and $p_{3}(T)$ have the same cyclic order in $\Gamma_{T}$ as $v_{1}, v_{2}$ and $v_{3}$ in $\partial B$. On the other hand, the curves $\gamma_{i}$ have no common points except $x$ because they are minimal geodesics 
in $M$. Then the Jordan curve theorem implies that their initial velocities $\gamma_{1}^{\prime}(0), \gamma_{2}^{\prime}(0)$ and $\gamma_{3}^{\prime}(0)$ in $U T_{x} M$ are cyclically ordered in the same way as the points $p_{1}, p_{2}$ and $p_{3}$ in $\Gamma_{T}$. The lemma follows.

We are now in a position to prove Conjecture A for the two-dimensional case. Choose a (sufficiently fine) cyclically ordered collection of linear functions $h_{1}, h_{2}, \ldots, h_{N} \in \partial B^{*}$. All indices below are taken modulo $N$. Let $F_{i}: M \rightarrow \mathbf{R}$ be their calibrating functions as in Proposition 4.3. For $i=1, \ldots, N$, define a linear map $L_{i}: V \rightarrow \mathbf{R}^{2}$ and a (Lipschitz) map $G_{i}: M \rightarrow \mathbf{R}^{2}$ by $L_{i}(v)=\left(h_{i}(v), h_{i+1}(v)\right)$ and $G_{i}(x)=\left(F_{i}(x), F_{i+1}(x)\right)$. Consider a measurable 2-form $\omega_{i}=d F_{i} \wedge d F_{i+1}$ on $M$. This form is the pull-back under $G_{i}$ of the Euclidean area form in $\mathbf{R}^{2}$, and it is $\mathbf{Z}^{2}$-periodic, so it can be thought of as a 2 -form on $M / \mathbf{Z}^{2}$. We want to compute the integral of $\omega$ over $M / \mathbf{Z}^{2}$. Observe that $G_{i}$ is equivariant with respect to the given action of $\mathbf{Z}^{2}$ on $M$ and the action of $L_{i}\left(\mathbf{Z}^{2}\right)$ on $\mathbf{R}^{2}$, and that the quotient map from $M / Z^{2}$ to a flat torus $\mathbf{R}^{2} / L_{i}\left(\mathbf{Z}^{2}\right)$ has degree 1 . Hence the integral of $\omega$ over $M / \mathbf{Z}^{2}$ equals the integral of the Euclidean area form over $\mathbf{R}^{2} / L_{i}\left(\mathbf{Z}^{2}\right)$, thus

$$
\int_{M / \mathbf{Z}^{2}} d F_{i} \wedge d F_{i+1}=m_{2}\left(\mathbf{R}^{2} / L_{i}\left(\mathbf{Z}^{2}\right)\right)
$$

where $m_{2}$ is the two-dimensional area coming from $\mathbf{R}^{2}$.

A value of a 2 -form in $M$ at a point $x \in M$ is a bi-vector in the vector space $T_{x}^{*} M$. Such bi-vectors can be represented by oriented areas of subsets of $T_{x}^{*} M$. In particular, the 2 -form $\frac{1}{2} d F_{i} \wedge d F_{i+1}$ is represented by the area of the triangle with vertices $0, d_{x} F_{i}$ and $d_{x} F_{i+1}$, taken with the appropriate sign. The above Lemma 5.1 implies that the co-vectors $\left\{d_{x} F_{i}\right\}_{i=1}^{N}$ are cyclically ordered in $U T_{x}^{*} M$, hence the sum (over all $i$ ) of the areas of these triangles equals the area of their convex hull. Thus

$$
\frac{1}{2} \sum_{i=1}^{N} m_{2}\left(\mathbf{R}^{2} / L_{i}\left(\mathbf{Z}^{2}\right)\right)=\int_{M / \mathbf{Z}^{2}} \sum_{i=1}^{N} \operatorname{area}\left(\operatorname{conv}\left\{d_{x} F_{1}, \ldots, d_{x} F_{N}\right\}\right) d x
$$

where $\operatorname{area}(\ldots) d x$ denotes the differential form represented by a given subset of $T^{*} M$. Denote by $B_{x}^{*} M$ the unit co-tangent ball at an $x \in M$, i. e. $B_{x}^{*} M=\left\{w \in T_{x}^{*} M: \Phi^{*}(w) \leq 1\right\}$. Recall that the two-dimensional HolmesThompson volume is given by

$$
\operatorname{Vol}_{2}\left(M / \mathbf{Z}^{2}\right)=\frac{1}{\pi} \int_{M / \mathbf{Z}^{2}} \operatorname{area}\left(B_{x}^{*}\right) d x .
$$

Since the $d_{x} F_{i}$ are unit co-vectors, their convex hull is contained in the unit ball, and we conclude that

$$
\frac{1}{2} \sum_{i=1}^{N} m_{2}\left(\mathbf{R}^{2} / L_{i}\left(\mathbf{Z}^{2}\right)\right) \leq \int_{M / \mathbf{Z}^{2}} \operatorname{area}\left(B_{x}^{*} M\right) d x=\pi \operatorname{Vol}\left(M / \mathbf{Z}^{2}, \Phi\right) .
$$


The same computation for the flat Banach space $(V,\|\cdot\|)$ with functions $h_{i}$ in place of $F_{i}$ yields

$$
\frac{1}{2} \sum_{i=1}^{N} m_{2}\left(\mathbf{R}^{2} / L_{i}\left(\mathbf{Z}^{2}\right)\right)=(1-\sigma) \pi \operatorname{Vol}\left(V / \mathbf{Z}^{2},\|\cdot\|\right)
$$

where $\sigma$ is defined by

$$
\operatorname{area}\left(\operatorname{conv}\left\{h_{1}, \ldots, h_{N}\right\}\right)=(1-\sigma) \operatorname{area}\left(B^{*}\right) .
$$

Thus $\operatorname{Vol}\left(M / \mathbf{Z}^{2}, \Phi\right) \geq(1-\sigma) \operatorname{Vol}\left(V / \mathbf{Z}^{2},\|\cdot\|\right)$. Since $\sigma$ can be made arbitrarily small, it follows that $\operatorname{Vol}\left(M / \mathbf{Z}^{2}, \Phi\right) \geq \operatorname{Vol}\left(V / \mathbf{Z}^{2},\|\cdot\|\right)$. This is equivalent to Conjecture A.

\section{EXAMPLE}

The goal of this section is to prove Theorem 2, namely to construct a fourdimensional Banach space in which the (Holmes-Thompson) area functional is not elliptic over $\mathbf{Q}$. In other words, we are going to give an example of a singular chain with rational coefficients such that the area of the chain is strictly less than the area of an affine disc with the same boundary. We will not worry about smothness of Banach norms in our construction since an example with a non-smooth norm can be smoothened. Indeed, if an example can be constructed in a Banach space with a non-smooth norm, the same surface would give examples for all sufficiently close norms due to the continuity of area functional with respect to the Banach norm.

We will show that every Banach space from a certain class has non-elliptic 2 -volume. First let us give one explicit example of a space from this class.

Let $B^{*} \subset \mathbf{R}^{4}$ be the convex hull of a curve parameterized by

$$
(\sin t, \cos t, \sin 3 t, \cos 3 t), \quad t \in \mathbf{R} .
$$

This set $B^{*}$ is a compact symmetric convex body, hence it is a unit ball of some norm $\|\cdot\|^{*}$. Let $\|\cdot\|$ be the dual to norm $\|\cdot\|^{*}$. Then the 2-volume defined by $\|\cdot\|$ is not elliptic over $\mathbf{Q}$; more precisely, a disc contained in the first coordinate plane does not minimize 2 -volume in the class of rational chains.

Now we pass to the proof. First we begin with a computation that explains where Busemann's examples of "reversed" Inequality 5 come from. This computation will enable us to construct such examples in Banach spaces with certain additional properties.

6.1. Stokes' Formula for Holmes-Thompson Area. Let $X$ be a fourdimensional vector space, and let $X^{*}$ denote the dual space (of linear functions $X \rightarrow \mathbf{R}$ ). Two-dimensional oriented linear subspaces of $X$ will be called planes. For every plane $V \subset X$ there is a natural linear map from $X^{*}$ to $V^{*}$, namely the restriction operator $\left.f \mapsto f\right|_{V}$. We call this map the projection to $V^{*}$ and denote it by $\operatorname{Pr}_{V}$ (if one identifies each space with its dual via a scalar product in $X$, this map turns to an orthogonal projection). 
Denote by $K_{2}(X)$ the two-dimensional Grassmanian cone of $X$, that is the subset of $X \wedge X$ consisting of simple bi-vectors. For a nonzero bi-vector $W \in K_{2}(X)$ we denote by $P(W)$ the plane in $X$ spanned by $W$. Observe that $W$ canonically defines an area form in the dual plane $P(W)^{*}$; indeed, a bi-vector $W$ is a linear function on $P(W)^{*} \wedge P(W)^{*}$, or, equivalently, a bi-linear skew-symmetric function on $P(W)^{*}$. We denote by Area $W$ the Lebesgue measure on $P(W)^{*}$ determined by this area form.

For a unit bi-vector $W$ in a Euclidean space, Area $W$ is just the standard Euclidean area in $P(W)^{*} \simeq P(W)$. One has Area ${ }_{\lambda W}=\lambda$ Area $_{W}$ for every $\lambda>0$.

Let $\|\cdot\|$ be a Banach norm on $X$, and $B^{*} \subset X^{*}$ be the unit ball of the dual norm. First we express the symplectic area $\mathrm{Vol}_{2}$ in $(X,\|\cdot\|)$ in terms of projections of $B^{*}$. We represent this area as a positively homogeneous function on the Grassmanian cone $K_{2}(X) \subset X \wedge X$. Namely, $\operatorname{Vol}_{2}(W)$ for a $W \in K_{2}(X)$ equals the area of a parallelogramm spanned by $W$. Then we have

$$
\operatorname{Vol}_{2}(W)=\frac{1}{\pi} \operatorname{Area}_{P(W)}\left(\operatorname{Pr}_{P(W)}\left(B^{*}\right)\right),
$$

i. e., our functional (on unit bi-vectors or just planes in a Euclidean space) is given by areas of projections of the convex body $B^{*}$. Indeed, for a plane $P \subset X$, the dual unit ball of the restriction of $\|\cdot\|$ to $P$ equals $\operatorname{Pr}_{P}\left(B^{*}\right)$, then the above formula follows from the definition of $\mathrm{Vol}_{2}$ (cf. formula 3).

One can integrate positively homogeneous functions on $K_{2}(X)$ over singular 2-chains in $X$, and integrating the above function $\mathrm{Vol}_{2}$ yields the symplectic area of a surface. Our goal is to compare $\mathrm{Vol}_{2}$ (for a suitable $\left.B^{*}\right)$ with a linear function $L$ on $X \wedge X$ restricted to $K_{2}(X)$. Note that the intergral of a linear function over a chain depends only on the chain's boundary because a linear function on $X \wedge X$ is nothing but a 2-form in $X$ with constant coefficients.

Fix a plane $V \subset X$. Now we restrict ourselves to bodies $B^{*}$ of special form. Namely we assume that $B^{*}$ is the convex hull of (the image of) a $C^{2}$-smooth curve $\gamma: S^{1} \rightarrow X^{*}$ such that the projection $\operatorname{Pr}_{V}$ o $\gamma$ is a simple, regular, right oriented and strictly convex closed curve in $V^{*}$. To simplify notation, we use the same letter $\gamma$ for the map and its image.

Now define a function $L: X^{*} \wedge X^{*} \rightarrow \mathbf{R}$ by

$$
L(W)=\frac{1}{2 \pi} \int_{S^{1}}\left(\gamma(t) \wedge \gamma^{\prime}(t)\right) \cdot W d t
$$

where the dot, of course, denotes the application of a 2-form to a bi-vector. Obviously $L$ is linear. Let $W \in K_{2}(X)$ and let $P=P(W)$ be the plane spanned by $W$. Since

$$
\left(w_{1} \wedge w_{2}\right) \cdot W=\left(\operatorname{Pr}_{P}\left(w_{1}\right) \wedge \operatorname{Pr}_{P}\left(w_{2}\right)\right) \cdot W
$$

for all $w_{1}, w_{2} \in X^{*}$, we have

$$
\left(\gamma(t) \wedge \gamma^{\prime}(t)\right) \cdot W=\left(\left(\operatorname{Pr}_{P} \circ \gamma\right)(t) \wedge\left(\operatorname{Pr}_{P} \circ \gamma\right)^{\prime}(t)\right) \cdot W .
$$


Note that the area form defined by $W$ on the dual plane $P^{*}=P(W)^{*}$ is nothing but multiplication of a 2-form by $W$. So one can interpet the last expression as the oriented area Area $W$ spanned by a vector in the projected curve $\operatorname{Pr}_{P} \circ \gamma$ and its velocity. Hence the quantity

$$
\pi L(W)=\int_{S^{1}} \frac{1}{2}\left(\left(\operatorname{Pr}_{P} \circ \gamma\right) \wedge\left(\operatorname{Pr}_{P} \circ \gamma\right)^{\prime}\right) \cdot(W)
$$

equals the area of the region(s) bounded by the projected curve $\operatorname{Pr}_{P} \circ \gamma$, counted with respective multiplicity (how many times the curve surrounds a region). In particular, if the projection is a simple and right oriented curve, then $\pi L(W)$ is the area of the region that it bounds. If the plane $P=P(W)$ is sufficiently close to our fixed plane $V$, then the projection is simple, right oriented and convex, so

$$
\pi L(W)=\operatorname{Area}_{W}\left(\operatorname{Conv}\left(\operatorname{Pr}_{P}(\gamma)\right)\right)=\operatorname{Area}_{W}\left(\operatorname{Pr}_{P}\left(B^{*}\right)\right)=\pi \operatorname{Vol}_{2}(W) .
$$

Thus we obtain the following

Lemma 6.1. $L=\mathrm{Vol}_{2}$ for all planes that are sufficiently close to $V$.

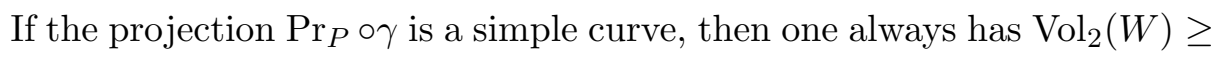
$L(W)$ because the area of a region in not greater than the area of its convex hull. However if the projection has self-intersections, some parts of the convex hull may be surrounded by the curve more than once (counted with multiplicity greater than one). As a result, it may happen that $\mathrm{Vol}_{2}<L$ for some planes. For instance, in the example given in the beginning of this section, the projection of the curve to the last coordinate plane is the triplycovered circle and therefore $L=3 \mathrm{Vol}_{2}>\mathrm{Vol}_{2}$ on this plane. We summarize our observatons in the following

Lemma 6.2. There exists a norm $\|\cdot\|$ on $X=\mathbf{R}^{4}=\{(x, y, z, t)\}$ and a linear function $L: X^{*} \wedge X^{*}$ such that $\mathrm{Vol}_{2}=L$ for all planes in a neighborhood of the xy-plane and $\mathrm{Vol}_{2}<L$ for the zt-plane.

6.2. A chain of small area. We will show that a norm from Lemma 6.2 is not elliptic over $\mathbf{Q}$. More precisely, we will construct a polyhedral surface $\beta$, parameterized by a planar polygon, such that its boundary $\partial \beta$ wraps $n$ times around the boundary of a region $A$ in the $x y$-plane, but $\operatorname{Vol}_{2}(\beta)<$ $n \cdot \operatorname{Vol}_{2}(A)$, for some natural number $n$. Define a function $\delta: K_{2}(X) \rightarrow \mathbf{R}$ by $\delta(W)=\operatorname{Vol}_{2}(W)-L(W)$. Then the desired inequality is equivalent to that

$$
\int_{\beta} \delta=\operatorname{Vol}_{2}(\beta)-\int_{\beta} L<0
$$

because the integral of $L$ over a chain $\beta$ depends only on the chain's boundary, and in our case equals $n \int_{A} L=n \operatorname{Vol}_{2}(A)$.

Fix an $\alpha>0$ such that $\mathrm{Vol}_{2}=L$ for all planes that form angles less than $3 \alpha$ with the $x y$-plane (here we use the first property from Lemma 6.2).

First we describe the triangulation of out surface. Let $n$ be sufficiently large. Let $a_{1} b_{1} a_{2} b_{2} \ldots a_{3 n} b_{3 n}$ be a regular $(6 n)$-gon in the plane centered 
at the origin $O$, and let $c_{1} \ldots c_{3 n}$ be a large regular $(3 n)$-gon obtained from $a_{1} \ldots a_{3 n}$ via a homothety centered at $O$. We triangulate the polygon $Q=c_{1} \ldots c_{3 n}$ into the polygon $a_{1} \ldots a_{3 n}$ and the triangles $a_{i} b_{i} a_{i+1}$, $a_{i} c_{i} b_{i}, b_{i} c_{i+1} a_{i+1}$ and $b_{i} c_{i} c_{i+1}$ (all indices are taken modulo $3 n$ ). We will construct the desired surface as a map $\beta: \mathbf{R}^{4}$ which is affine in each element of this triangulation.

For the vertices $a_{i}$, define $\beta\left(a_{i}\right)=p_{i}$ where $p_{1} \ldots p_{3 n}$ is a regular polygon in the $z t$-plane such that $\left|p_{i}\right|=1$ for all $i$. Let $\varepsilon(n)=\left|p_{i} p_{i+1}\right|$, the side of this polygon (measured in the Euclidean metric).

Let $v_{1}, v_{2}, v_{3}$ be three unit vectors in the $x y$-plane forming angles $2 \pi / 3$ with each other. Define

$$
\beta\left(b_{i}\right)=q_{i}:=\frac{p_{i}+p_{i+1}}{2}+\frac{\varepsilon(n)}{\alpha} \cdot v_{i \bmod 3}
$$

for $i=1, \ldots, 3 n$. This defines the images of the triangles $a_{i} b_{i} a_{i+1}$; they are thin equilateral triangles whose lateral sides are almost parallel (form angles less than $\alpha$ ) to vectors $v_{1}, v_{2}, v_{3}$. Finally, choose a very large $R>0$ and define

$$
\beta\left(c_{i}\right)=r_{i}:=R \cdot \frac{v_{(i-1) \bmod 3}+v_{i \bmod 3}}{2} .
$$

This determines the images of all vertices and thus defines the map $\beta: Q \rightarrow$ $\mathbf{R}^{4}$. Observe that the $\beta$-image of the boundary $\partial Q$ wraps $n$ times around the triangle $A=\triangle r_{1} r_{2} r_{3}$ which is contained in the $x y$-plane.

It remains to prove that $\int_{\beta} \delta<0$ provided that $n$ and $R$ are large enough. First observe that the triangles containing "distant" points $r_{i}=\beta\left(c_{i}\right)$ are almost parallel to the $x y$-plane in the sense that their two-dimensional oriented directions are $(3 \varepsilon)$-close to the $x y$-plane (if $R=\left|r_{i}\right|$ is no less than, say, $10 / \varepsilon$ ). Therefore $\delta=0$ for these triangles (by lemma 6.1 , hence the integral $\int_{\beta} \delta$ is composed of the integral over the polygon $p_{1} \ldots p_{3 n}$ and the integrals over the triangles $p_{i} q_{i} p_{i+1}$. The former is negative and bounded away from zero (as $n \rightarrow \infty)$ since $\delta<0$ for the $z t$-plane and the area of the polygon converges to a positive constant. On the other hand, the total area of the triangles $p_{i} q_{i} p_{i+1}$ goes to zero as $n \rightarrow \infty$, so does the corresponding term in the integral of $\delta$. Thus for a large enough $n$, the negative contribution to $\int_{\beta} \delta$ coming from integration over the polygon $p_{1} \ldots p_{3 n}$ outweigths the possible positive contribution coming from integration over the triangles, and therefore $\int_{\beta} \delta<0$. This completes the proof of Theorem 4 .

\section{REFERENCES}

[Al] J.-C. Alvarez Paiva "Some problems in Finsler Geometry", preprint, 23 pages

[Ba] V. Bangert "Geodesic rays, Busemann functions and monotone twist maps" Calc. Var., vol 2, 1994, 49-63

[Be] A. Besicovitch "On two problems of Loewner" J. London Math. Soc., vol 27, 1952, pp 141-144 
[BCG] G. Besson, G. Courtois, S. Gallot "Entropies et rigidités des espaces localement symétriques de courbure strictement négative", Geom. Funct. Anal., vol 5, issue 5, 1995, pp. 731-799

[BI1] D. Burago, S. Ivanov "On Asymptotic Volume of Tori". "Geometry and Functional Analysis", vol 5, 1995, pp. 800-808

[BI2] D. Burago, S. Ivanov "Isometric Embeddings of Finsler Manifolds", St. Petersburg Math Journal, vol 5, 1994, pp 159-169

[BuZ] Yu. Burago and V. Zalgaller "Geometric inequalities". Springer-Verlag, 1988

[Bu1] D. Burago, "Periodic metrics" Advances in Soviet Math. New York vol 9, 1992, 205-210

$[\mathrm{Bu}]$ H. Busemann "The Geometry of Geodesics", Acad. Press, New York, 1955

[BES] H. Busemann, G. Ewalds, and S. Shephard "Convex bodies and convexity on Grassmann cones. I-IV", Math. Ann. vol 151, 1963 pp. 1-41

[Cr] C. B. Croke "Rigidity and the distance between boundary points", J. Differential Geometry vol 33, 1991, pp. 445-464

[Gr] M. Gromov "Filling Riemannian manifolds", J. Diff. Geom. vol 18, 1983 pp. 1-147

[Iv] S. Ivanov "On two-dimensional minimal fillings", St. Petersburg Math Journal, to appear, 2000.

$[\mathrm{Pu}] \mathrm{P} . \mathrm{Pu}$ "Some inequalities in certain non-orientable Riemannian manifolds", Pacific J. Math. vol 2, 1952, pp. 55-71

[Sa] L. A. Santaló "Integral geometry and geometric probability", Encyclopedia Math. Appl. Addison-Wesley, London, 1976

[SW] R. Schnieder, J. A. Wiecker "Integral Geometry in Minkowski Spaces", Advances in Mathematics, vol 129, 1997, pp. 222-260

[Th] A. C. Thompson "Minkowski Geometry", Encyclopedia of Math and Its Applications, Vol. 63, Cambridge Univ. Press. 1996

Department of Mathematics, The Pennsylvania State University, UniverSITY PARK, PA 16802

E-mail address: burago@math.psu.edu

E-mail address: svivanov@pdmi.ras.ru 